\title{
Center of mass of a system of variable mass particles
}

\author{
M.T. Thomaz ${ }^{*}[$ E.V. Corrêa Silva 20 \\ ${ }^{1}$ Universidade Federal Fluminense, Instituto de Física, Niterói, RJ, Brasil \\ ${ }^{2}$ Universidade do Estado do Rio de Janeiro, Faculdade de Tecnologia, Departamento de Matemática, Física e Computação, \\ Resende, RJ, Brasil
}

Received on December 10, 2018; Accepted on January 11, 2019.

\begin{abstract}
We study the dynamics of the center of mass (CM) of a set of particles when the number of particles in the system varies during a short interval of time, or when the value of the mass of one of the particles depends on time. We show that in both situations, while the population or mass of the system varies, the linear momentum of the CM differs from the total linear momentum of the system. During this time interval, the dynamics of the $\mathrm{CM}$ is driven by the external forces that act on the system plus transient forces that depend on the position and velocity of the CM. This dynamics is applied to study the time evolution of the vector position of the CM of a system of free particles moving on an air rail in which the mass of one the particles varies in a short time interval. We want to show it to students that with the knowledge and tools they have at an elementary Mechanics course they can formulate and answer questions which are not in the textbooks.

Keywords: Classical Mechanics, center of mass, linear momentum, variable mass, variable number of particles, one-dimensional motion.
\end{abstract}

\section{Introduction}

The concept of the center of mass (CM) of a system with fixed number $N \geq 2$ of particles with constant masses is commonly approached in introductory courses of Mechanics $1-6$. To the CM of the system one associates a fictitious particle, with mass chosen to be equal to the sum of the masses of the particles in the set. The dynamics of the CM is driven by the external forces that act on the $N$ particles. The kinematics of the fictitious particle located at the CM of the system, for instance, its position, velocity and acceleration vectors, are obtained from the respective physical quantities of the particle that belong to the system.

Although the position of the CM of this type of set of particles may not coincide with the position of any of the physical particles of the set, the CM of the system has interesting properties. For instance, the total weight forces that act on the particles of a rigid body has the same effect on the motion of the body as it would if the resultant of those forces were applied on the CM. An important physical quantity associated to the CM is its linear momentum, that is equal to the total linear momentum of the system of particles. The equality of these two vectors are very important in the study of particle collision, and their conservation is helpful for the study of the elementary Particle Physics inside atoms, nuclei and nucleons.
Understanding the dynamics of systems with variable mass is of great relevance, for instance, to the study of ship and rocket motions $7-10$. In the present paper we characterize the $\mathrm{CM}$ of a particle system in two different scenarios: $a$ ) when the number of particles in the system varies by one unit, and this transition happens in a very short time, but all particle masses are constant; $b$ ) when the number of particles in the system is fixed, but the mass of one of them varies in time. We study the dynamics of the CM of a system of particles in both scenarios and determine the form of Newton's Second Law for the dynamics of the CM during the whole movement, including the transition time interval of each scenario. Our aim is that of relating, in each of those systems, the linear momentum of the CM to the total linear momentum of system at any time $t$. We point out that outside the transition time interval, we have the same conditions in the system as is usually described in the literature and the known results are recovered from ours. It is certainly interesting to ask questions that go beyond what is traditionally presented in textbooks which may possibly lead to new knowledge, regardless of how experimentally feasible those possibilities might currently be.

In section 2 we describe the kinematics of a set of $N$ fixed particles that is split in two subsystems: $S_{1}$ and $S_{2}$. The masses of all particles are constant. A given particle, the $L$-th particle, migrates from $S_{1}$ to $S_{2}$ during a short transition time $\Delta t$. In subsection $2 \mathrm{~A}$ we relate the linear momentum of the CM of subsystems $S_{1}$, and

\footnotetext{
*Endereço de correspondência: mtt@if.uff.br
} 
$S_{2}$ to the total linear momentum of their respective particles, for $t \in(-\infty, \infty)$. In subsection $2 \mathrm{~B}$ we derive the dynamics of the CM of $S_{1}$, including the transition time interval when the $L$-th particle moves from $S_{1}$ to $S_{2}$. In section 3 we deal with a single system with $N$ particles in which one of those particles has time-dependent mass. The treatment is analogous to that of section 2 , but the resulting equation of motion for the CM differs slightly. In section 4 we show the behavior of a simple example of a unidimensional system consisting of three free particles sliding on an air rail, in which one of them has variable mass. In section 5 we present our conclusions. In Appendix A we calculate at any time $t$ the position of the CM of a system with $N$ particles, $N \geq 2$, in any scenario, when the number of particles can vary with time and the value of their masses can depend on time. In Appendix B we present a particular function that has the same properties as the transition function $f(t)$.

\section{The case of two subsystems with varying number of particles}

\subsection{Kinematics}

Consider a classical system $S$ of $N$ particles with constant masses $\left\{m_{1}, m_{2}, \ldots, m_{N}\right\}$. Its center of mass (CM) has a position vector given by

$$
\vec{R}_{C M}(t)=\frac{m_{1} \vec{r}_{1}(t)+m_{2} \vec{r}_{2}(t)+\cdots+m_{N} \vec{r}_{N}(t)}{m_{1}+m_{2}+\cdots+m_{N}},
$$

in which $m_{i}$ and $\vec{r}_{i}(t)$ are the mass and the position of the $i$-th particle at time $t$, respectively, for $i \in\{1,2, \ldots, N\}$. A fictitious particle is assumed to coincide with the position of the CM of the system of the physical particles. The mass of this fictitious particle is chosen to be equal to the value of the total mass of the $N$ particles. We assume an inertial frame of reference, in which all measurements are made. In a [possibly non-inertial] frame of reference solidary to the CM, the system total momentum is zero [1]. The kinematics of the center of mass (CM) stems from the kinematics of the particles that compose the system.

\subsubsection{Subsystems, permanent particles and the migrating particle}

Let $S$ be decomposed into two subsystems $S_{1}$ and $S_{2}$ so that that the first $(L-1)$ particles, in which $L$ is an integer and $1<L<N$, always belong to $S_{1}$ - let us call them the permanent particles of $S_{1}$. In the same token, the last $(N-L)$ particles are the permanent particles of the subsystem $S_{2}$. For the remaining $L$-th particle - let it be called the migrating particle - suppose that there is some transition time interval $T=\left[t_{1}, t_{2}\right]$ so that such particle belong to $S_{1}$ for all $t<t_{1}$ and belong to $S_{2}$ for all $t>t_{2}$. During that interval, which can be written as

$$
T=\left[\bar{t}-\frac{\Delta t}{2}, \quad \bar{t}+\frac{\Delta t}{2}\right]
$$

in terms of its amplitude

$$
\Delta t=\left(t_{2}-t_{1}\right)>0
$$

and its mid-point

$$
\bar{t}=\frac{t_{1}+t_{2}}{2},
$$

the migrating $L$-th particle crosses the boundary from $S_{1}$ to $S_{2}$ (say, due to the particle and/or the boundary motions).

In order to have non-singular kinematic functions defined and the total number of physical particles in the set $S$ equal to $N$ at any time $t$, consider some smooth real transition function $f(t)$ that has the following properties:

$$
f(t)=\left\{\begin{array}{l}
0, \text { for } t<-\frac{\Delta t}{2} \\
\frac{1}{2}+h(t) \in[0,1], \text { for } t \in\left[-\frac{\Delta t}{2}, \frac{\Delta t}{2}\right], \\
1, \quad \text { for } t>\frac{\Delta t}{2},
\end{array}\right.
$$

so that $h(t)$ is an odd smooth function, $h(-t)=-h(t)$. Consequently,

$$
f(-t)=\left\{\begin{array}{l}
1, \text { for } t<-\frac{\Delta t}{2} \\
\frac{1}{2}-h(t) \in[0,1], \text { for } t \in\left[-\frac{\Delta t}{2}, \frac{\Delta t}{2}\right] \\
0, \text { for } t>\frac{\Delta t}{2},
\end{array}\right.
$$

and

$$
f(t)+f(-t)=1
$$

for all $t$. The value of the time interval $\Delta t$ determines the rapidity of the transition of the $L$-th particle from the subsystem $S_{1}$ to the other subsystem $\left(S_{2}\right)$. In Appendix $\mathrm{B}$ we present an explicit example of a function that satisfies conditions (5). With $f(t)$ defined as such, the functions

$$
N_{1}(t) \equiv L-f(t-\bar{t})
$$

and

$$
\begin{aligned}
N_{2}(t) & =N-N_{1}(t)=N-L+f(t-\bar{t}) \\
& =N-L+1-f(\bar{t}-t),
\end{aligned}
$$

can be interpreted (at least for $t \notin T$ ) as the number of particles in subsystems $S_{1}$ and $S_{2}$, respectively. Before the transition, which occurs while $t \in\left[\bar{t}-\frac{\Delta t}{2}, \bar{t}+\frac{\Delta t}{2}\right]$, where $\Delta t$ and $\bar{t}$ are given by $(3)$ and $(\sqrt{4})$, respectively, we have $N_{1}=L$ and $N_{2}=N-L$; after the transition, we have $N_{1}=L-1$ and $N_{2}=N-L+1$.

For our present purposes, we do not need to specify $f(x)$ any further. For a particular realization of the function $f(t)$, the reader is referred to Appendix B. 
Our aim is that of obtaining Newton's Second Law for the CM of a subsystem like $S_{1}$ or $S_{2}$, with varying number of particles, within a larger system with total constant number of particles. All particles have constant masses. In what follows, we will obtain expressions for the kinematics of the fictitious particles whose locations coincide with the CM of those subsystems, and relate them to the kinematics of the physical particles that belong to these subsystems, that is, $S_{1}$ and/or $S_{2}$.

\subsubsection{The masses and centers of mass of the subsystems $S_{1}$ and $S_{2}$}

Analogous to (1), the position of the $\mathrm{CM}$ of $S_{1}$ is written as

$$
\vec{R}_{C M}^{(1)}(t)=\frac{\sum_{i=1}^{L-1} m_{i} \vec{r}_{i}(t)+m_{L} f(\bar{t}-t) \vec{r}_{L}(t)}{\sum_{i=1}^{L-1} m_{i}+m_{L} f(\bar{t}-t)},
$$

whereas the position of the CM of $S_{2}, \vec{R}_{C M}^{(2)}(t)$ is

$$
\vec{R}_{C M}^{(2)}(t)=\frac{m_{L} f(t-\bar{t}) \vec{r}_{L}(t)+\sum_{i=L+1}^{N} m_{i} \vec{r}_{i}(t)}{m_{L} f(t-\bar{t})+\sum_{i=L+1}^{N} m_{i}} .
$$

The masses of the subsystems $S_{1}$ and $S_{2}$ at any time $t$ are

$$
M_{1}(t)=m_{1}+m_{2}+\cdots+m_{L-1}+m_{L} f(\bar{t}-t)
$$

and

(13)

$$
M_{2}(t)=m_{L} f(t-\bar{t})+m_{L+1}+\cdots+m_{N-1}+m_{N},
$$

respectively.

The mass of the fictitious particle at the CM of the subsystem $S_{1}, \vec{R}_{C M}^{(1)}(t)$, is chosen to be equal to $M_{1}(t)$, and the mass of the fictitious particle at the CM of the subsystem $S_{2}$ is also chosen to be equal to the sum of masses of the physical particles that belong to $S_{2}$ at every time $t t^{1}$

The total mass $M$ of the system $S$ is constant,

$$
\begin{aligned}
M & =M_{1}(t)+M_{2}(t) \\
& =\sum_{i=1}^{L-1} m_{i}+m_{L} \underbrace{[f(\bar{t}-t)+f(t-\bar{t})]}_{=1}+\sum_{j=L+1}^{N} m_{j} \\
& =\sum_{i=1}^{N} m_{i} .
\end{aligned}
$$

One can rewrite 10 and 111 as

$$
M_{1}(t) \vec{R}_{C M}^{(1)}(t)=\sum_{i=1}^{L-1} m_{i} \vec{r}_{i}(t)+m_{L} f(\bar{t}-t) \vec{r}_{L}(t)
$$

and

$$
M_{2}(t) \vec{R}_{C M}^{(2)}(t)=m_{L} f(t-\bar{t}) \vec{r}_{L}(t)+\sum_{i=L+1}^{N} m_{i} \vec{r}_{i}(t)
$$

and then obtain

$$
\begin{aligned}
& M_{1}(t) \vec{R}_{C M}^{(1)}(t)+M_{2}(t) \vec{R}_{C M}^{(2)}(t)=\sum_{i=1}^{L-1} m_{i} \vec{r}_{i}(t) \\
& +m_{L} \underbrace{[f(\bar{t}-t)+f(t-\bar{t})]}_{=1} \vec{r}_{L}+\sum_{i=L+1}^{N} m_{i} \vec{r}_{i}(t) \\
& =\sum_{i=1}^{N} m_{i} \vec{r}_{i}(t)
\end{aligned}
$$

which, according to (1) and (14), equals to

$$
M_{1}(t) \vec{R}_{C M}^{(1)}(t)+M_{2}(t) \vec{R}_{C M}^{(2)}(t)=M \vec{R}_{C M}(t) .
$$

The velocities of each CM of the total system $S$ and the subsystems $S_{1}$ and $S_{2}$ are, respectively,

$$
\begin{aligned}
& \vec{V}_{C M}(t)=\frac{d \vec{R}_{C M}(t)}{d t}, \\
& \vec{V}_{C M}^{(1)}(t)=\frac{d \vec{R}_{C M}^{(1)}(t)}{d t}, \\
& \vec{V}_{C M}^{(2)}(t)=\frac{d \vec{R}_{C M}^{(2)}(t)}{d t} .
\end{aligned}
$$

One should readily notice that

$$
\vec{V}_{C M}^{(1)} \neq \frac{1}{M(t)}[\sum_{i=1}^{L-1} m_{i} \vec{v}_{i}(t)+\underbrace{m_{L}(t)}_{m_{L} f(\bar{t}-t)} \vec{v}_{L}(t)] .
$$

In fact, by differentiating 10, , one obtain ${ }^{2}$

$$
\begin{aligned}
& \vec{V}_{C M}^{(1)}=\frac{1}{M(t)}\left[\sum_{i=1}^{L-1} m_{i} \vec{v}_{i}(t)+m_{L} f(\bar{t}-t) \vec{v}_{L}(t)+\right. \\
& \left.+m_{L} \frac{d f(\bar{t}-t)}{d t} \vec{r}_{L}(t)\right]-\frac{1}{M(t)^{2}} \frac{d M(t)}{d t}\left[\sum_{i=1}^{L-1} m_{i} \vec{r}_{i}(t)\right. \\
& \left.+m_{L} f(\bar{t}-t) \vec{r}_{L}(t) .\right] .
\end{aligned}
$$

In order to relate the linear momentum of the CM of the system $S$ and those of the subsystems $S_{1}$ and $S_{2}$, we differentiate (18), obtaining

$$
\begin{aligned}
& \frac{d M_{1}(t)}{d t} \vec{R}_{C M}^{(1)}(t)+M_{1}(t) \vec{V}_{C M}^{(1)}(t) \\
& +\frac{d M_{2}(t)}{d t} \vec{R}_{C M}^{(2)}(t)+M_{2}(t) \vec{V}_{C M}^{(2)}(t)=M \vec{V}_{C M}(t) .
\end{aligned}
$$

\footnotetext{
${ }^{1}$ If the system is under a constant gravitational acceleration $\vec{g}$, the total weight forces of the particles that constitute the subsystems $S_{1}$ and $S_{2}$ can be considered to be applied in their centers of mass, located at $\vec{R}_{C M}^{(1)}(t)$ and $\vec{R}_{C M}^{(2)}(t)$, respectively.

${ }^{2}$ During the transition time interval $T$, the expression of $V_{C M}^{(i)}, i \in\{1,2\}$, is different from that of a set particles with constant mass.
} 
As

$$
\frac{d f(\bar{t}-t)}{d t}=\frac{d}{d t}(1-f(t-\bar{t}))=-\frac{d f(t-\bar{t})}{d t},
$$

from (12), 13) and (7), one obtains

$$
\begin{aligned}
\frac{d M_{1}(t)}{d t} & =m_{L} \frac{d f(\bar{t}-t)}{d t}=-m_{L} \frac{d f(t-\bar{t})}{d t} \\
\frac{d M_{2}(t)}{d t} & =m_{L} \frac{d f(t-\bar{t})}{d t}
\end{aligned}
$$

\subsubsection{The relation between the linear} momentum of the CM of the system $S$ and the CM's of the subsystems $S_{1}$ and $S_{2}$

The relation (24) can be written as

$$
\begin{aligned}
& M_{1}(t) \vec{V}_{C M}^{(1)}(t)+M_{2}(t) \vec{V}_{C M}^{(2)}(t) \\
& =M \vec{V}_{C M}(t)+m_{L} \frac{d f(t-\bar{t})}{d t}\left(\vec{R}_{C M}^{(1)}(t)-\vec{R}_{C M}^{(2)}(t)\right)
\end{aligned}
$$

or

$$
\begin{aligned}
& \vec{P}_{C M}^{(1)}(t)+\vec{P}_{C M}^{(2)}(t)=\vec{P}_{C M}(t)+ \\
& +m_{L} \frac{d f(t-\bar{t})}{d t}\left(\vec{R}_{C M}^{(1)}(t)-\vec{R}_{C M}^{(2)}(t)\right),
\end{aligned}
$$

in which

$$
\begin{gathered}
\vec{P}_{C M}^{(1)}(t) \equiv M_{1}(t) \vec{V}_{C M}^{(1)}(t), \\
\vec{P}_{C M}^{(2)}(t) \equiv M_{2}(t) \vec{V}_{C M}^{(2)}(t)
\end{gathered}
$$

are the linear momenta of the CM of $S_{1}$ and $S_{2}$, respectively, whereas

$$
\vec{P}_{C M}(t) \equiv M \vec{V}_{C M}(t),
$$

in which $M$ is the total mass (14) of the CM of the $N$ physical particles and $\vec{P}_{C M}(t)$ is the linear momentum of the CM of the whole system $S$. Observe that the sum of $\vec{P}_{C M}^{(1)}(t)$ and $\vec{P}_{C M}^{(2)}(t)$ does not add up to $\vec{P}_{C M}(t)$ during the transition interval (2). The linear momenta $\vec{P}_{C M}^{(1)}(t)$ and $\vec{P}_{C M}^{(2)}(t)$ recover the usual definition of the momenta of each subsystem for times $t$ outside the transition interval $T$ in which the $L$-th particle migrates from $S_{1}$ to $S_{2}$, as discussed in the next subsection.
The linear momentum of the $i$-th particle is

$$
\vec{p}_{i}(t)=m_{i} \vec{v}_{i}(t)=m_{i} \frac{d \vec{r}_{i}(t)}{d t},
$$

for $i \in\{1,2, \ldots, N\}$. The total linear momentum $\vec{P}(t)$ of the whole system, sum of all individual linear momenta, corresponds, as expected, to the linear momentum of (the fictitious particle at) the $\mathrm{CM}$ of the system $S$ :

$$
\begin{aligned}
\vec{P}(t) & =\sum_{i=1}^{N} \vec{p}_{i}(t)=\sum_{i=1}^{N} m_{i} \frac{d \vec{r}_{i}(t)}{d t} \\
& =\frac{d}{d t} \sum_{i=1}^{N} m_{i} \vec{r}_{i}(t)=\frac{d}{d t}\left(M \vec{R}_{C M}(t)\right) \\
& =M \vec{V}_{C M}(t)
\end{aligned}
$$

which, according to 32 , leads to

$$
\vec{P}(t)=\vec{P}_{C M}(t),
$$

showing that the linear momentum of the CM of $S$ is equal to the total liner momentum of the set of the $N$ physical particles.

\subsubsection{The linear momentum of a subsystem is NOT ALWAYS equal to the total linear momenta of its physical particles}

A relation analogous to that of 35 does not hold for all $t$, however, for the subsystems $S_{1}$ and $S_{2}$. Let us examine, for instance, the linear momentum of the CM of $S_{1}$, defined in (30), and compare it to the total momentum $\vec{P}^{(1)}(t)$ of subsystem $S_{1}$, which reads

$$
\begin{aligned}
\vec{P}^{(1)}(t) & =\vec{p}_{1}(t)+\vec{p}_{2}(t)+\cdots+\vec{p}_{L-1}(t)+f(\bar{t}-t) \vec{p}_{L}(t) \\
& =\sum_{k=1}^{L-1} \vec{p}_{k}(t)+f(\bar{t}-t) \vec{p}_{L}(t) .
\end{aligned}
$$

Eq. 10 is used to relate the vector $\vec{P}_{C M}^{(1)}(t)$ and the information about the physical particles that belong to the subsystem $S_{1}$ at each moment,

$$
\begin{aligned}
\vec{P}_{C M}^{(1)}(t) & =M_{1}(t) \vec{V}_{C M}^{(1)}(t)=M_{1}(t) \frac{d \vec{R}_{C M}^{(1)}(t)}{d t} \\
& =M_{1}(t) \frac{d}{d t}\left\{\frac{1}{M_{1}(t)}\left[m_{1} \vec{r}_{1}(t)+m_{2} \vec{r}_{2}(t)+\cdots+m_{L-1} \vec{r}_{L-1}(t)+m_{L} f(\bar{t}-t) \vec{r}_{L}(t)\right]\right\} .
\end{aligned}
$$


So,

$$
\begin{aligned}
\vec{P}_{C M}^{(1)}(t)= & M_{1}(t)\left\{-\frac{1}{M_{1}(t)^{2}} \frac{d M_{1}(t)}{d t}\left[\sum_{i=1}^{L-1} m_{i} \vec{r}_{i}(t)+m_{L} f(\bar{t}-t) \vec{r}_{L}(t)\right]\right. \\
& \left.+\frac{1}{M_{1}(t)}\left[\sum_{i=1}^{L-1} m_{i} \frac{d \vec{r}_{i}(t)}{d t}+m_{L}\left(\frac{d f(\bar{t}-t)}{d t} \vec{r}_{L}(t)+f(\bar{t}-t) \frac{d \vec{r}_{L}(t)}{d t}\right)\right]\right\} .
\end{aligned}
$$

This can be rewritten due to $(26)$ as

$$
\begin{aligned}
\vec{P}_{C M}^{(1)}(t)= & \frac{m_{L}}{M_{1}(t)} \frac{d f(t-\bar{t})}{d t}\left[m_{1} \vec{r}_{1}(t)+m_{2} \vec{r}_{2}(t)+\cdots+m_{L-1} \vec{r}_{L-1}(t)+m_{L} f(\bar{t}-t) \vec{r}_{L}(t)\right] \\
& +\vec{p}_{1}(t)+\vec{p}_{2}(t)+\cdots+\vec{p}_{L-1}(t)+f(\bar{t}-t) \vec{p}_{L}(t)+m_{L} \frac{d f(\bar{t}-t)}{d t} \vec{r}_{L}(t)
\end{aligned}
$$

We simplify (39) by using (15) and 25),

$$
\vec{P}_{C M}^{(1)}(t)=m_{L} \frac{d f(t-\bar{t})}{d t} \vec{R}_{C M}^{(1)}(t)+\left[\sum_{i=1}^{L-1} \vec{p}_{i}(t)+f(\bar{t}-t) \vec{p}_{L}(t)\right]-m_{L} \frac{d f(t-\bar{t})}{d t} \vec{r}_{L}(t),
$$

which amounts to

$$
\vec{P}_{C M}^{(1)}(t)=\vec{P}^{(1)}(t)+m_{L} \frac{d f(t-\bar{t})}{d t}\left(\vec{R}_{C M}^{(1)}(t)-\vec{r}_{L}(t)\right) .
$$

We use 30 to rewrite $V_{C M}^{(1)}$ in terms of the linear momenta of the particles that belong to the subset $S_{1}$,

$$
\vec{V}_{C M}^{(1)}(t)=\frac{\vec{p}_{1}(t)+\vec{p}_{2}(t)+\cdots+\vec{p}_{L-1}(t)+f(\bar{t}-t) \vec{p}_{L}(t)}{M_{1}(t)}-\frac{m_{L}}{M_{1}(t)} \frac{d f(t-\bar{t})}{d t}\left(\vec{r}_{L}(t)-\vec{R}_{C M}^{(1)}(t)\right) .
$$

Unlike the case of the whole system stated in $(35)$, the linear momentum of the CM of the subsystem $S_{1}, \vec{P}_{C M}^{(1)}(t)$, is not equal to the total momentum $\vec{P}^{(1)}(t)$ of that subsystem during the interval of time that the function $f(t-\bar{t})$ varies between the values 0 and 1 . This interval of time corresponds to the transition of the $L$-th particle between the two subsystems $S_{1}$ and $S_{2}$.

An analogous statement can be found for the subsystem $S_{2}$, namely,

$$
\vec{P}_{C M}^{(2)}(t)=\vec{P}^{(2)}(t)-m_{L} \frac{d f(t-\bar{t})}{d t}\left(\vec{R}_{C M}^{(2)}(t)-\vec{r}_{L}(t)\right),
$$

relating the linear momentum of its $\mathrm{CM}$, as defined in [31, and the total momentum $\vec{P}^{(2)}(t)$ of the physical particles that belong to $S_{2}$, which reads

$$
\begin{aligned}
& \vec{P}^{(2)}(t)=f(t-\bar{t}) \vec{p}_{L}(t)+\vec{p}_{L+1}(t)+\ldots \\
& +\vec{p}_{N-1}(t)+\vec{p}_{N}(t) .
\end{aligned}
$$

By adding (41) and (43), one recovers (29).

\subsection{Dynamics of the CM of $S_{1}$}

\subsubsection{Equation of motion for the $\mathrm{CM}$ of the subsystem $S_{1}$}

The equation of motion of the $i$-th particle of the system $S$, observed from an inertial frame, can be written as

$$
\frac{d \vec{p}_{i}(t)}{d t}=\vec{F}_{i}^{(e x t)}(t)+\sum_{\substack{j=1 \\ j \neq i}}^{N} \vec{F}_{j \rightarrow i}^{(i n t)}(t)
$$

in which $\vec{F}_{i}^{(e x t)}(t)$ is the sum of the external forces acting on the $i$-th particle (that is, due to interactions with other bodies not belonging to the system $S$ ) and $\vec{F}_{j \rightarrow i}^{(i n t)}(t)$ is the internal force on the $i$-th particle due to its interaction with the $j$-th particle of $S$. The summation index $j$ runs over all particles of $S$, excluding the $i$-particle itself. Newton's Third Law can be expressed as

$$
\vec{F}_{i \rightarrow j}^{(i n t)}(t)=-\vec{F}_{j \rightarrow i}^{(i n t)}(t) .
$$

The equation of motion for the ficticious particle on the CM of subsystem $S_{1}$ is, from the time derivative of 41,

$$
\begin{aligned}
& \frac{d \vec{P}_{C M}^{(1)}(t)}{d t}=m_{L} \frac{d^{2} f(t-\bar{t})}{d t^{2}}\left(\vec{R}_{C M}^{(1)}(t)-\vec{r}_{L}(t)\right) \\
& +m_{L} \frac{d f(t-\bar{t})}{d t}\left(\vec{V}_{C M}^{(1)}(t)-\vec{v}_{L}(t)\right)+\frac{d \vec{P}^{(1)}(t)}{d t} \\
& =\vec{F}_{R}^{(C M, 1)}(t) .
\end{aligned}
$$


Equality (48) is Newton's Second Law for a variable mass particle. $\vec{F}_{R}^{(C M, 1)}(t)$ is the resulting force acting on the fictitious particle located in the CM of $S_{1}$ so that it follows the path $\vec{R}_{C M}^{(1)}(t)$ as its mass changes with time.

One is reminded that $M_{1} \vec{V}_{C M}^{(1)}(t)=\vec{P}_{C M}^{(1)}(t)$ and $m_{L} \vec{v}_{L}(t)=\vec{p}_{L}(t)$, the linear momentum of the CM of $S_{1}$ and of the migrating $L$-th particle, respectively. The expression of the vector $\vec{P}^{(1)}(t)$ is given by 36 . Ob- serve that the r.h.s of this equation contains not only the expected (last) term, the time derivative of the total momentum of the physical particles that belong to the subsystem $S_{1}$, but also terms on the first and second time derivatives of the transition function $f(t-\bar{t})$. Hence, for a well defined equation of motion for the CM of this subsystem, a continuous function $f(t)$ with continuous derivatives in the variable $t$ up to second order would suffice.

\subsubsection{Rewriting $\frac{d \vec{P}^{(1)}(t)}{d t}$ in terms of internal and external forces applied to $S_{1}$}

Let us rewrite 47 in terms of the forces acting on the particles of the system. First, let us observe that its last term is equivalent to

$$
\frac{d \vec{P}^{(1)}(t)}{d t}=\sum_{k=1}^{L-1} \frac{d \vec{p}_{k}(t)}{d t}+\frac{d f(\bar{t}-t)}{d t} \vec{p}_{L}(t)+f(\bar{t}-t) \frac{d \vec{p}_{L}(t)}{d t}
$$

by using (36). From the individual equations of motion (45), comes

$$
\frac{d \vec{P}^{(1)}(t)}{d t}=\sum_{k=1}^{L-1}\left[\vec{F}_{k}^{(e x t)}(t)+\sum_{\substack{j=1 \\ j \neq k}}^{N} \vec{F}_{j \rightarrow k}^{(i n t)}(t)\right]+\frac{d f(\bar{t}-t)}{d t} \vec{p}_{L}(t)+f(\bar{t}-t)\left[\vec{F}_{L}^{(e x t)}(t)+\sum_{\substack{j=1 \\ j \neq L}}^{N} \vec{F}_{j \rightarrow L}^{(i n t)}(t)\right] .
$$

By grouping the terms related to external and internal forces applied to the physical particles that belong to $S_{1}$, one obtains

$$
\frac{d \vec{P}^{(1)}(t)}{d t}=\left[\sum_{k=1}^{L-1} \vec{F}_{k}^{(e x t)}(t)+f(\bar{t}-t) \vec{F}_{L}^{(e x t)}(t)\right]+\left[\sum_{k=1}^{L-1} \sum_{\substack{j=1 \\ j \neq k}}^{N} \vec{F}_{j \rightarrow k}^{(i n t)}(t)+f(\bar{t}-t) \sum_{\substack{j=1 \\ j \neq L}}^{N} \vec{F}_{j \rightarrow L}^{(i n t)}(t)\right]-\frac{d f(t-\bar{t})}{d t} \vec{p}_{L}(t)
$$

in which once more we have used the result 25 on the derivative of the function $f(t)$.

We will focus our attention now on the double summation involving the internal forces. By splitting the inner summation in three parts, for $j<L, j=L$ and $j>L$, one obtains

$$
\begin{aligned}
\sum_{k=1}^{L-1} \sum_{\substack{j=1 \\
j \neq k}}^{N} \vec{F}_{j \rightarrow k}^{(i n t)}(t) & =\sum_{k=1}^{L-1}\left(\sum_{\substack{j=1 \\
j \neq k}}^{L-1} \vec{F}_{j \rightarrow k}^{(i n t)}(t)+\vec{F}_{L \rightarrow k}^{(i n t)}(t)+\sum_{j=L+1}^{N} \vec{F}_{j \rightarrow k}^{(i n t)}(t)\right) \\
& =\sum_{k=1}^{L-1} \sum_{\substack{j=1 \\
j \neq k}}^{L-1} \vec{F}_{j \rightarrow k}^{(i n t)}(t)+\sum_{k=1}^{L-1} \vec{F}_{L \rightarrow k}^{(i n t)}(t)+\sum_{k=1}^{L-1} \sum_{j=L+1}^{N} \vec{F}_{j \rightarrow k}^{(i n t)}(t) .
\end{aligned}
$$

(As $k \leq L-1$, the constraint $j \neq k$ is redundant for $j \geq L$, so it has been kept solely for the summation in which $j \leq L-1$.)

For the sake of mathematical simplicity only, and without any loss of generality, let us define $\vec{F}_{i \rightarrow i}^{(i n t)} \equiv \overrightarrow{0}$, for $i \in\{1,2, \ldots, N\}$ - which still satisfies Newton's Third Law (46), and let us relax about the (again redundant) condition $j \neq k$. We may then write the first term of $[52]$ as

$$
\begin{aligned}
\sum_{k=1}^{L-1} \sum_{\substack{j=1 \\
j \neq k}}^{L-1} \vec{F}_{j \rightarrow k}^{(i n t)}(t) & =\sum_{k=1}^{L-1} \sum_{j=1}^{L-1} \vec{F}_{j \rightarrow k}^{(i n t)}(t)=\frac{1}{2}\left[\sum_{k=1}^{L-1} \sum_{j=1}^{L-1} \vec{F}_{j \rightarrow k}^{(i n t)}(t)+\sum_{k=1}^{L-1} \sum_{j=1}^{L-1} \vec{F}_{j \rightarrow k}^{(i n t)}(t)\right] \\
& =\frac{1}{2}\left[\sum_{k=1}^{L-1} \sum_{j=1}^{L-1} \vec{F}_{j \rightarrow k}^{(i n t)}(t)+\sum_{j=1}^{L-1} \sum_{k=1}^{L-1} \vec{F}_{k \rightarrow j}^{(i n t)}(t)\right],
\end{aligned}
$$


in which we simply interchanged the dummy summation indices $\{j, k\}$ without altering the summation in the second term of (53). By using Newton's Third Law (46) and then swapping the summation order, one obtains

$$
\sum_{k=1}^{L-1} \sum_{\substack{j=1 \\ j \neq k}}^{L-1} \vec{F}_{j \rightarrow k}^{(i n t)}(t)=\frac{1}{2}\left[\sum_{k=1}^{L-1} \sum_{j=1}^{L-1} \vec{F}_{j \rightarrow k}^{(i n t)}(t)-\sum_{j=1}^{L-1} \sum_{k=1}^{L-1} \vec{F}_{j \rightarrow k}^{(i n t)}(t)\right]=\frac{1}{2}\left[\sum_{k=1}^{L-1} \sum_{j=1}^{L-1} \vec{F}_{j \rightarrow k}^{(i n t)}(t)-\sum_{k=1}^{L-1} \sum_{j=1}^{L-1} \vec{F}_{j \rightarrow k}^{(i n t)}(t)\right]=\overrightarrow{0}
$$

in which $\overrightarrow{0}$ is the null vector $(|\overrightarrow{0}|=0)$.

Hence 52$]$ is reduced to

$$
\sum_{k=1}^{L-1} \sum_{\substack{j=1 \\ j \neq k}}^{N} \vec{F}_{j \rightarrow k}^{(i n t)}(t)=\sum_{k=1}^{L-1} \vec{F}_{L \rightarrow k}^{(i n t)}(t)+\sum_{k=1}^{L-1} \sum_{j=L+1}^{N} \vec{F}_{j \rightarrow k}^{(i n t)}(t)
$$

and consequently 51 now reads

$$
\begin{aligned}
\frac{d \vec{P}^{(1)}(t)}{d t} & =\left[\sum_{k=1}^{L-1} \vec{F}_{k}^{(e x t)}(t)+f(\bar{t}-t) \vec{F}_{L}^{(e x t)}(t)\right]+\left[\sum_{k=1}^{L-1} \vec{F}_{L \rightarrow k}^{(i n t)}(t)+f(\bar{t}-t) \sum_{\substack{j=1 \\
j \neq L}}^{N} \vec{F}_{j \rightarrow L}^{(i n t)}(t)+\sum_{k=1}^{L-1} \sum_{j=L+1}^{N} \vec{F}_{j \rightarrow k}^{(i n t)}(t)\right] \\
& -\frac{d f(t-\bar{t})}{d t} \vec{p}_{L}(t) .
\end{aligned}
$$

But

$$
\begin{aligned}
& \sum_{k=1}^{L-1} \vec{F}_{L \rightarrow k}^{(i n t)}(t)+f(\bar{t}-t) \sum_{\substack{j=1 \\
j \neq L}}^{N} \vec{F}_{j \rightarrow L}^{(i n t)}(t)=\sum_{k=1}^{L-1} \vec{F}_{L \rightarrow k}^{(i n t)}(t)+f(\bar{t}-t)\left[\sum_{j=1}^{L-1} \vec{F}_{j \rightarrow L}^{(i n t)}(t)+\sum_{j=L+1}^{N} \vec{F}_{j \rightarrow L}^{(i n t)}(t)\right] \\
& =\sum_{k=1}^{L-1} \vec{F}_{L \rightarrow k}^{(i n t)}(t)+f(\bar{t}-t)\left[-\sum_{j=1}^{L-1} \vec{F}_{L \rightarrow j}^{(i n t)}(t)+\sum_{j=L+1}^{N} \vec{F}_{j \rightarrow L}^{(i n t)}(t)\right]=(1-f(\bar{t}-t)) \sum_{k=1}^{L-1} \vec{F}_{L \rightarrow k}^{(i n t)}(t) \\
& +f(\bar{t}-t) \sum_{j=L+1}^{N} \vec{F}_{j \rightarrow L}^{(i n t)}(t)=f(t-\bar{t}) \sum_{k=1}^{L-1} \vec{F}_{L \rightarrow k}^{(i n t)}(t)+f(\bar{t}-t) \sum_{k=L+1}^{N} \vec{F}_{k \rightarrow L}^{(i n t)}(t) .
\end{aligned}
$$

Thus 56 can be rewritten as

$$
\begin{aligned}
\frac{d \vec{P}^{(1)}(t)}{d t} & =\left[\sum_{k=1}^{L-1} \vec{F}_{k}^{(e x t)}(t)+f(\bar{t}-t) \vec{F}_{L}^{(e x t)}(t)\right]+\left[f(t-\bar{t}) \sum_{k=1}^{L-1} \vec{F}_{L \rightarrow k}^{(i n t)}(t)+f(\bar{t}-t) \sum_{k=L+1}^{N} \vec{F}_{k \rightarrow L}^{(i n t)}(t)\right. \\
& \left.+\sum_{k=1}^{L-1} \sum_{j=L+1}^{N} \vec{F}_{j \rightarrow k}^{(i n t)}(t)\right]-\frac{d f(t-\bar{t})}{d t} \vec{p}_{L}(t) .
\end{aligned}
$$

Inserting (58) into 47) yields

$$
\begin{aligned}
\frac{d \vec{P}_{C M}^{(1)}(t)}{d t} & =m_{L} \frac{d^{2} f(t-\bar{t})}{d t^{2}}\left(\vec{R}_{C M}^{(1)}(t)-\vec{r}_{L}(t)\right)+m_{L} \frac{d f(t-\bar{t})}{d t}\left(\vec{V}_{C M}^{(1)}(t)-\vec{v}_{L}(t)\right) \\
& +\left[\sum_{k=1}^{L-1} \vec{F}_{k}^{(e x t)}(t)+f(\bar{t}-t) \vec{F}_{L}^{(e x t)}(t)\right]+\left[f(t-\bar{t}) \sum_{k=1}^{L-1} \vec{F}_{L \rightarrow k}^{(i n t)}(t)+f(\bar{t}-t) \sum_{k=L+1}^{N} \vec{F}_{k \rightarrow L}^{(i n t)}(t)\right. \\
& \left.+\sum_{k=1}^{L-1} \sum_{j=L+1}^{N} \vec{F}_{j \rightarrow k}^{(i n t)}(t)\right]-\frac{d f(t-\bar{t})}{d t} \vec{p}_{L}(t)
\end{aligned}
$$


or

$$
\begin{aligned}
\frac{d \vec{P}_{C M}^{(1)}(t)}{d t} & =m_{L} \frac{d^{2} f(t-\bar{t})}{d t^{2}}\left(\vec{R}_{C M}^{(1)}(t)-\vec{r}_{L}(t)\right)+m_{L} \frac{d f(t-\bar{t})}{d t}\left(\vec{V}_{C M}^{(1)}(t)-2 \vec{v}_{L}(t)\right) \\
& +\left[\sum_{k=1}^{L-1} \vec{F}_{k}^{(e x t)}(t)+f(\bar{t}-t) \vec{F}_{L}^{(e x t)}(t)\right]+\left[f(t-\bar{t}) \sum_{k=1}^{L-1} \vec{F}_{L \rightarrow k}^{(i n t)}(t)+f(\bar{t}-t) \sum_{k=L+1}^{N} \vec{F}_{k \rightarrow L}^{(i n t)}(t)\right. \\
& \left.+\sum_{k=1}^{L-1} \sum_{j=L+1}^{N} \vec{F}_{j \rightarrow k}^{(i n t)}(t)\right] \\
& =\vec{F}_{R}^{(C M, 1)}(t) .
\end{aligned}
$$

That is the equation of motion for the CM of $S_{1}$, in terms of variables related to the center of mass itself $\left(\vec{R}_{C M}^{(1)}, \vec{P}_{C M}^{(1)}\right)$, to the migrating $L$-th particle $\left(\vec{r}_{L}, \vec{p}_{L}\right)$, to the forces acting on each particle of $S_{1}$ due to the particles in $S_{2}$ besides the external forces of $S$ that act on the physical particles belonging to $S_{1}$ during the whole movement, that is $t \in(-\infty,+\infty)$. Notice that during the transition time in which the L-th particle moves from $S_{1}$ to $S_{2}$, its motion is affected by the internal forces acting on it, modulated by the transition function $f(t)$.

Eq. 61 relates the effective force $\vec{F}_{R}^{(C M, 1)}(t)$ that acts on the fictitious particle located at the CM of $S_{1}$, and the forces applied to the physical particles belonging to the subset $S_{1}$. Besides the physical forces contributing to $\vec{F}_{R}^{(C M, 1)}(t)$ we have the contribution of the migrating particle $L$, responsible for the time variation of the mass of the fictitious particle, $M_{1}(t)$.

\subsubsection{The equation of motion of the CM of $S_{1}$ in terms of its acceleration, masses and external forces to $S_{1}$}

Defining the acceleration of the CM of the subsystem $S_{1}$ as

$$
\vec{a}_{C M}^{(1)}(t) \equiv \frac{d \vec{V}_{C M}^{(1)}(t)}{d t}
$$

and taking $(26)$ into account, we may rewrite the l.h.s. of 60 as

$$
\begin{aligned}
& \frac{d \vec{P}_{C M}^{(1)}(t)}{d t}=\frac{d}{d t}\left(M_{1}(t) \vec{V}_{C M}^{(1)}(t)\right)=\frac{d M_{1}(t)}{d t} \vec{V}_{C M}^{(1)}(t)+M_{1}(t) \frac{d \vec{V}_{C M}^{(1)}(t)}{d t}=-m_{L} \frac{d f(t-\bar{t})}{d t} \vec{V}_{C M}^{(1)}(t) \\
& +M_{1}(t) \vec{a}_{C M}^{(1)}(t) \Rightarrow M_{1}(t) \vec{a}_{C M}^{(1)}(t)=\frac{d \vec{P}_{C M}^{(1)}(t)}{d t}+\frac{d f(t-\bar{t})}{d t} m_{L} \vec{V}_{C M}^{(1)}(t) . \\
& =\vec{F}_{R}^{(C M, 1)}(t)+\frac{d f(t-\bar{t})}{d t} \cdot m_{L} \vec{V}_{C M}^{(1)}(t) .
\end{aligned}
$$

By inserting this equality into $(60)$ and isolating the acceleration term, we obtain the equation of motion for the CM of the subsystem $S_{1}$,

$$
\begin{aligned}
M_{1}(t) \vec{a}_{C M}^{(1)}(t) & =m_{L} \frac{d^{2} f(t-\bar{t})}{d t^{2}}\left(\vec{R}_{C M}^{(1)}(t)-\vec{r}_{L}(t)\right)+2 m_{L} \frac{d f(t-\bar{t})}{d t}\left(\vec{V}_{C M}^{(1)}(t)-\vec{v}_{L}(t)\right) \\
& +\left[\sum_{k=1}^{L-1} \vec{F}_{k}^{(e x t)}(t)+f(\bar{t}-t) \vec{F}_{L}^{(e x t)}(t)\right]+\left[f(t-\bar{t}) \sum_{k=1}^{L-1} \vec{F}_{L \rightarrow k}^{(i n t)}(t)+f(\bar{t}-t) \sum_{k=L+1}^{N} \vec{F}_{k \rightarrow L}^{(i n t)}(t)\right. \\
& \left.+\sum_{k=1}^{L-1} \sum_{j=L+1}^{N} \vec{F}_{j \rightarrow k}^{(i n t)}(t)\right]
\end{aligned}
$$

valid for $t \in(-\infty,+\infty)$. Alternatively, from $(26)$ we have

$$
\begin{aligned}
\frac{d f(t-\bar{t})}{d t} & =-\frac{1}{m_{L}} \frac{d M_{1}(t)}{d t}, \\
\frac{d^{2} f(t-\bar{t})}{d t^{2}} & =-\frac{1}{m_{L}} \frac{d^{2} M_{1}(t)}{d t^{2}}
\end{aligned}
$$


and (65) amounts to

$$
\begin{aligned}
M_{1}(t) \vec{a}_{C M}^{(1)}(t) & =\left[-\frac{d^{2} M_{1}(t)}{d t^{2}}\left(\vec{R}_{C M}^{(1)}(t)-\vec{r}_{L}(t)\right)-2 \frac{d M_{1}(t)}{d t}\left(\vec{V}_{C M}^{(1)}(t)-\vec{v}_{L}(t)\right)\right] \\
& +\left[\sum_{k=1}^{L-1} \vec{F}_{k}^{(e x t)}(t)+f(\bar{t}-t) \vec{F}_{L}^{(e x t)}(t)\right]+\left[f(t-\bar{t}) \sum_{k=1}^{L-1} \vec{F}_{L \rightarrow k}^{(i n t)}(t)+f(\bar{t}-t) \sum_{k=L+1}^{N} \vec{F}_{k \rightarrow L}^{(i n t)}(t)\right. \\
& \left.+\sum_{k=1}^{L-1} \sum_{j=L+1}^{N} \vec{F}_{j \rightarrow k}^{(i n t)}(t)\right] .
\end{aligned}
$$

The "particle" that is placed at the CM of $S_{1}$ is a fictitious one. During the transition time interval in which the $L$-th particle migrates from $S_{1}$ to $S_{2}$, the internal forces that act on the $L$-th particle contribute to the acceleration $\vec{a}_{C M}^{(1)}(t)$.

\subsubsection{Interpreting the force terms in the dynamics of the CM of $S_{1}$}

All the physical forces on the r.h.s. of (68) that contribute to the dynamics of the $\mathrm{CM}$ of $S_{1}$ are forces that act, at time $t$, on the physical particles that belong to this subsystem. When the number of particles in each subsystem does not vary in time, $\frac{d M_{1}(t)}{d t}=0$, we recover Newton's Second Law, that rules the time variation of the vector position of the fictitious particle located at the $\mathrm{CM}$ of the set of the physical particles.

The three bracketed groups of terms in the r.h.s. of equation (68) account for distinct contributions to the dynamics of the CM of the subsystem $S_{1}$ : the first from the time variation of its mass $M_{1}(t)$; the second from the external forces acting on its permanent particles and on the migrating particle; and the last one from the internal forces (of the system as a whole), but they are internal or external forces that act on the subsystem $S_{1}$ depending on the different intervals of time, that is, $t<\bar{t}-\frac{\Delta t}{2}$, $t \in\left[\bar{t}-\frac{\Delta t}{2}, \bar{t}+\frac{\Delta t}{2}\right]$ and $t>\bar{t}+\frac{\Delta t}{2}$. For $t<\bar{t}+\frac{\Delta t}{2}$ the $L$-th particle belongs to $S_{1}$ and the forces in the second term in the third bracket are external to $S_{1}$. For $t>\bar{t}+\frac{\Delta t}{2}$ the $L$-th particle does not belong to $S_{1}$ and the first set of forces in the third bracket are external forces to $S_{1}$ and the second set of forces in the same bracket does not act on any particle of subsystem $S_{1}$.

The term

$$
-\frac{d^{2} M_{1}(t)}{d t^{2}}\left(\vec{R}_{C M}^{(1)}(t)-\vec{r}_{L}(t)\right)
$$

on the r.h.s. of (68) can be interpreted as fictitious restoration or repelling force, depending on the sign of the coefficient $\frac{d^{2} M_{1}(t)}{d t^{2}}$, acting on the CM, whereas the term

$$
-2 \frac{d M_{1}(t)}{d t}\left(\vec{V}_{C M}^{(1)}(t)-\vec{v}_{L}(t)\right)
$$

is a fictitious viscous damping force. Both forces are due to the mass variation of $S_{1}$, being non-vanishing only during the transition interval. (They are proportional to derivatives of the transition function (5), which vanish outside this interval.)
The term

$$
\sum_{k=1}^{L-1} \vec{F}_{k}^{(e x t)}(t)
$$

represents the external forces to the system, acting on the permanent particles of $S_{1}$, whereas

$$
\sum_{k=1}^{L-1} \sum_{j=L+1}^{N} \vec{F}_{j \rightarrow k}^{(i n t)}(t),
$$

represents those external forces exchanged between the permanent particles of $S_{1}$ and the permanent particles of $S_{2}$.

We can say that the migrating $L$-th particle is no longer in $S_{1}$ after the transition interval is over $t>\bar{t}+\frac{\Delta t}{2}$. With that in mind, we can interpret the remaining terms. Before and during the transition interval, that is $t \in\left(-\infty, \bar{t}+\frac{\Delta t}{2}\right]$, the term

$$
f(\bar{t}-t) \vec{F}_{L}^{(e x t)}(t)
$$

accounts for the external force on the migrating particle while it belongs to $S_{1}$ as we see from the conditions (5) that defines the function $f(t)$. The term

$$
f(\bar{t}-t) \sum_{k=L+1}^{N} \vec{F}_{k \rightarrow L}^{(i n t)}(t)
$$

on the r.h.s. of 68 describes the interaction of the permanent particles of the other subsystem $S_{2}$ with the migration particle while it belongs to $S_{1}$, which corresponds to external forces to $S_{1}$. During and after the transition, the term

$$
f(t-\bar{t}) \sum_{k=1}^{L-1} \vec{F}_{L \rightarrow k}^{(i n t)}(t)
$$

depicts the interaction of the migrating particle and the permanent particles of $S_{1}$ during the transition and after the $L$-th particle has left the subsystem $S_{1}$. These forces are internal to the set of particles composing $S_{1}$ for $t<\bar{t}+\frac{\Delta t}{2}$ and external otherwise.

The results 71 - 74 show which forces in the subsystem $S_{1}$ account for the movement of the CM of $S_{1}$, 
besides the forces $(69)$ and $(70)$ due to the variation of the total number of physical particles belonging to $S_{1}$.

Notice that, according to Eq. (68), during time interval in which the $L$-th particle goes from $S_{1}$ to $S_{2}$, we may have $\vec{a}_{C M}^{(1)} \neq 0$ even if all $N$ particles of the system are free (with $\vec{F}_{k}^{(e x t)}=\overrightarrow{0}$ and $\vec{F}_{k \rightarrow L}^{(i n t)}=\overrightarrow{0}$, for all $l, k \in\{1,2, \ldots, N\})$. Even though observed from an inertial reference frame, the fictitious particle placed at the CM of $S_{1}$ may have non-null acceleration, even if all physical forces that act on the particles of $S_{1}$ vanish.

\subsection{A simple example}

As a simple realization of a particle set where the number of particles varies in time, we consider two free particles that slide on a horizontal air rail. The two particles have the same value $m$ of mass. They leave from the same position $x=0$ at $t=0 \mathrm{~s}$, but they slide in opposite directions in their one-dimensional motion along the air rail.

The positions and the velocities of the particles $1, x_{1}(t)$ and $v_{1}(t)$, and $2, x_{2}(t)$ and $v_{2}(t)$, are

$$
\begin{aligned}
& x_{1}(t)=t \times\left(\frac{m}{s}\right) \Rightarrow v_{1}(t)=1 \times\left(\frac{m}{s}\right), \\
& x_{2}(t)=-t \times\left(\frac{m}{s}\right) \Rightarrow v_{1}(t)=-1 \times\left(\frac{m}{s}\right) .
\end{aligned}
$$

Initially both particles belong to the set $S$. For $t \gtrsim$ $3.25 \mathrm{~s}$ particle 2 leaves this particle set. To describe this transition of particle 2 , we choose the function $f(3 s-t)$, see eq.(B9),

$$
f(3 s-t)=\frac{1}{2}[1+\tanh (10(3 s-t))] .
$$

Following 10 , we define the position of the CM of the set $S$ to be

$$
X_{C M}(t) \equiv \frac{m x_{1}(t)+f(3 s-t) \cdot m x_{2}(t)}{m+f(3 s-t) m} .
$$

The mass of the fictitious particle located at the CM of $S$ is given by 12 with $L=2$, that is,

$$
\begin{aligned}
M_{C M}(t) & =m+f(3 s-t) m \\
& =\frac{m}{2}[3+\tanh (10(3 s-t))] .
\end{aligned}
$$

Substituting the expressions of $x_{i}(t)$, with $i=1,2$, and the transition function $f(3 s-t)$ on the r.h.s. of 79 , we obtain the position of the CM of $S$ during the whole movement,

$$
X_{C M}(t)=t \cdot \frac{[1-\tanh (10(3 s-t))]}{\frac{1}{2}[3+\tanh (10(3 s-t))]} .
$$

In Fig.17 we plot the positions of the particles 1 and 2 (while the latter remains in the set $S$ ), and the CM of $S$. For $t \lesssim 3.25 \mathrm{~s}$ the CM of $S$ remains at the origin $x=0$, since both particles have the same mass, they travel in opposite directions and with the same modulus of velocity. During the transition time the location of the CM of $S$ goes from $x=0$ up to the position of particle 1 . After particle 2 leaves $S$, the position of the CM coincides with the position of particle 1 as we can see in Fig.1.1.

The velocity of the CM of $S, V_{C M}(t)$, is the time variation of $X_{C M}(t)$, that is

$$
V_{C M}(t)=\frac{d X_{C M}(t)}{d t}=\frac{1+40 t-2 \sinh (10(3 s-t)) \cdot \cosh (10(3 s-t))+2 \cosh ^{2}(10(3 s-t))}{[3 \cosh (10(3 s-t))+\sinh (10(3 s-t))]^{2}} .
$$

Fig $1 \mathrm{~b}$ shows the velocities of the two particles while they belong to the set $S$ and the CM of the latter. Before $t=2.75 \mathrm{~s}$, when particle 2 begins to leave $S$, the CM of the particle set remains at rest. During the interval of time when particle 2 migrates from $S$, the velocity of the CM suffers a sudden variation in time up it reaches the velocity of particle 1 , that is kept constant during the whole movement.

Finally the dynamics of the fictitious particle located at the CM of $S$ is driven by Newton's second law for a particle with varying mass, $M_{C M}(t)$,

$$
\frac{d}{d t}\left[M_{C M}(t) V_{C M}(t)\right]=F_{R}(t),
$$

where $F_{R}(t)$ is the resultant force applied to the fictitious particle at the CM.

Substituting the expressions 81 and 83 on the 1.h.s. of 84 we can derive the expression of the force $F_{R}(t)$ after a simple but tedious calculation.

Fig.1 1 represents the curve $F_{R}(t) \times t$ and shows that the movement of the fictitious particle at the CM has a free evolution before and after the transition interval of time when particle 2 leaves $S$. During the time interval $t \in(2.75 s, 3.25 s)$ a non null force $F_{R}(t)$ acts on the fictitious particle. In this example we consider the case when in eqs. 60 and (61) we do not have the contributions of physical forces to the motion of the two particles.

\section{Center of mass of a system with of particles with variable mass}

In this section we will consider a system of $N$ physical particles of masses $\left\{m_{1}, m_{2}, \ldots m_{N}\right\}$, in which $N \geq 2$, so that the $N$-th particle has a variable mass $m_{N}(t)$. The movement of this set of particles will be observed from 
an inertial frame of reference (in which Newton's Second Law is valid),

$$
\frac{d \vec{p}_{i}(t)}{d t}=\frac{d}{d t}\left(m_{i}(t) \vec{v}_{i}(t)\right)=\sum_{\substack{j=1 \\ j \neq i}}^{N} \vec{F}_{j \rightarrow i}^{(i n t)}(t)+\vec{F}_{i}^{(e x t)}(t)
$$
in which $i \in\{1,2, \ldots, N\}, \vec{r}_{i}(t)$ is the position of the $i$-th particle, $m_{i}$ its mass - fixed for all particles, $i \in\{1,2, \ldots N-1\}$ but for the $N$-th one, $m_{N}=m_{N}(t)$ - and $\vec{v}_{i}(t)$ is its velocity. $\vec{p}_{i}(t)$ is the linear momentum of the $i$-th particle, $\vec{F}_{j \rightarrow i}^{(i n t)}$ is the (internal) force that the $j$-th particle applies on it, whereas $\vec{F}_{i}^{(e x t)}$ is the resultant of all external forces applied to that $i$-th particle. We point out that for the $N$-th particle, the one whose mass varies in time, the physical forces that act on it are responsible for the time variation of the product $m_{N}(t) \cdot \vec{v}_{N}(t)$, whereas the physical forces that act on the other $N-1$ particles in the set only drive the time dependence of their respective vector velocities.

Nowadays we do not know physical (non-relativistic) particles whose masses vary in time. However, this should not stop us from asking questions about the Physics that governs particles with varying mass; it is part of the role of Theoretical Physics presenting challenges for Experimental Physics. Moreover, one is reminded that a "particle" can be understood as any physical body whose dimensions are ignored for the sake of a given context.

The position of the CM of this set of $N$ particles is defined as

$$
\vec{R}_{C M}(t) \equiv \frac{m_{1} \vec{r}_{1}(t)+m_{2} \vec{r}_{2}(t)+\cdots+m_{N}(t) \vec{r}_{N}(t)}{M_{C M}(t)},
$$

in which

$$
M_{C M}(t) \equiv m_{1}+m_{2}+\cdots+m_{N}(t)
$$

is the mass of the fictitious particle whose position coincides with the location CM at any time $t$, and this mass is equal to the total mass of the system, similar to (1). The time dependence of $\vec{R}_{C M}(t)$ comes not only from the movement of particles itself (i.e., from the trajectories $\left.\vec{r}_{i}(t)\right)$, but also from the time dependence of the mass of the $N$-th particle. Using 86 as a definition for the CM position of the set formed by the $N$ particles, the location for the application of their total weight remains in $\vec{R}_{C M}(t)$.

To the ficticious particle located at the CM we assign a linear momentum given by

$$
\vec{P}_{C M}(t)=M_{C M}(t) \vec{V}_{C M}(t),
$$

in which $\vec{V}_{C M}(t)$ is the CM velocity,

$$
\begin{aligned}
\vec{V}_{C M}(t) & =\frac{d \vec{R}_{C M}(t)}{d t}=\frac{d}{d t}\left[\frac{m_{1} \vec{r}_{1}(t)+m_{2} \vec{r}_{2}(t)+\cdots+m_{N}(t) \vec{r}_{N}(t)}{M_{C M}(t)}\right] \\
& =\frac{m_{1} \vec{v}_{1}(t)+m_{2} \vec{v}_{2}(t)+\cdots+m_{N}(t) \vec{v}_{N}(t)}{M_{C M}(t)} \\
& -\frac{1}{M_{C M}(t)^{2}} \frac{d M_{C M}(t)}{d t}\left[m_{1} \vec{r}_{1}(t)+m_{2} \vec{r}_{2}(t)+\cdots+m_{N}(t) \vec{r}_{N}(t)\right]+\frac{1}{M_{C M}(t)} \frac{d m_{N}(t)}{d t} \vec{r}_{N}(t)
\end{aligned}
$$
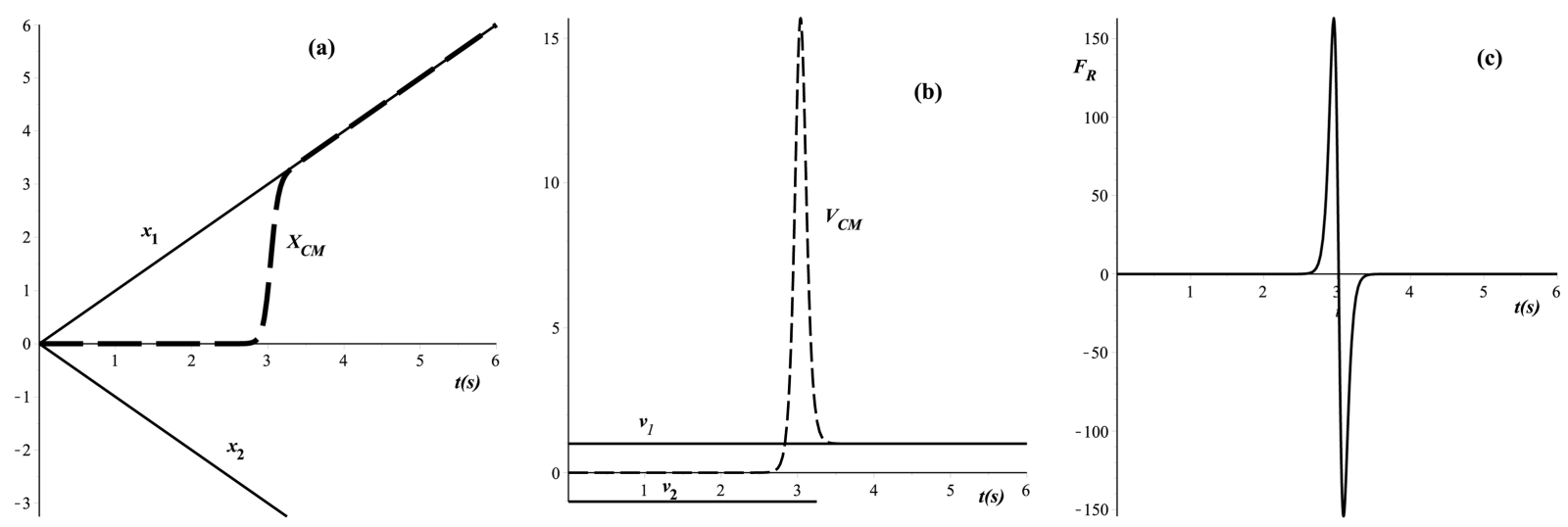

Figure 1: $a$ ) The position of the particles 1 and 2 (solid lines), and the CM (dashed line) of $S$ as functions of $t$. $b$ ) The time dependence of the velocities of the particles (solid lines) that belong to $S$ and its CM (dashed line). $c$ ) The curve of $F_{R} \times t, F_{R}(t)$ being the resultant force applied on the fictitious particle at the CM during the whole movement. 
However, from 86 ,

$$
-\frac{1}{M_{C M}(t)^{2}} \frac{d M_{C M}(t)}{d t}\left[m_{1} \vec{r}_{1}(t)+m_{2} \vec{r}_{2}(t)+\cdots+m_{N}(t) \vec{r}_{N}(t)\right]=-\frac{1}{M_{C M}(t)}\left(\frac{d M_{C M}(t)}{d t}\right) \vec{R}_{C M}(t),
$$

so that

$$
\vec{V}_{C M}(t)=\frac{m_{1} \vec{v}_{1}(t)+m_{2} \vec{v}_{2}(t)+\cdots+m_{N}(t) \vec{v}_{N}(t)}{M_{C M}(t)}+\frac{1}{M_{C M}(t)}\left[\frac{d m_{N}(t)}{d t} \vec{r}_{N}(t)-\frac{d M_{C M}(t)}{d t} \vec{R}_{C M}(t)\right] .
$$

From 87 we have

$$
\frac{d M_{C M}(t)}{d t}=\frac{d m_{N}(t)}{d t}
$$

which results in

$$
\vec{V}_{C M}(t)=\frac{m_{1} \vec{v}_{1}(t)+m_{2} \vec{v}_{2}(t)+\cdots+m_{N}(t) \vec{v}_{N}(t)}{M_{C M}(t)}+\frac{1}{M_{C M}(t)} \frac{d m_{N}(t)}{d t}\left[\vec{r}_{N}(t)-\vec{R}_{C M}(t)\right] .
$$

The first term in the r.h.s. of (93) is the ordinary result for a system of particles with constant mass. The second term accounts for the dependence of the velocity of the $\mathrm{CM}$ on the variation of the mass of the $N$-th particle. This previous result coincides with $(42)$ when $N=L$, and $m_{L}(t)=m_{L} \cdot f(\bar{t}-t)$, in which $m_{L}$ is a constant value and $f(\bar{t}-t)$ is the transition function described in subsection 2.1. To verify that both results are equal, we should notice that the coefficient of the last term on the r.h.s. of $(42)$ can be rewritten as

$$
-\frac{m_{L}}{M_{1}(t)} \frac{d f(t-\bar{t})}{d t}=\frac{1}{M_{1}(t)} \frac{d m_{L}(t)}{d t} .
$$

The linear momentum 88 then amounts to

$$
\vec{P}_{C M}(t)=m_{1} \vec{v}_{1}(t)+m_{2} \vec{v}_{2}(t)+\cdots+m_{N}(t) \vec{v}_{N}(t)+\frac{d m_{N}(t)}{d t}\left[\vec{r}_{N}(t)-\vec{R}_{C M}(t)\right]
$$

or

$$
\vec{P}_{C M}(t)=\vec{P}(t)+\frac{d m_{N}(t)}{d t}\left[\vec{r}_{N}(t)-\vec{R}_{C M}(t)\right]
$$

in which $\vec{P}(t)$ is the total linear momentum of the system,

$$
\vec{P}(t)=\vec{p}_{1}(t)+\vec{p}_{2}(t)+\cdots+\vec{p}_{N}(t)=\sum_{i=1}^{N} m_{i}(t) \vec{v}_{i}(t)
$$

Eq. (96) shows that the linear momentum of the CM and the total momentum of the system only coincide under at least one of two situations: when all particle masses are constant; or when the positions of the CM and of the particle with variable mass are the same for all $t$. Newton's Second Law for the fictitious particle located at the CM and with varying mass, $M_{C M}(t)$, is

$$
\begin{aligned}
\frac{d\left(\vec{P}_{C M}(t)\right)}{d t} & =\vec{F}_{R}^{(C M)}(t) \\
& =\frac{d\left(M_{C M}(t) \vec{V}_{C M}(t)\right)}{d t}
\end{aligned}
$$

in which $\vec{F}_{R}^{(C M)}(t)$ is the resulting force acting on the fictitious particle located at the CM of the set of $N$ particles.

The equation of motion for the fictitious particle at the CM is obtained by differentiating (96), and taking (88) into account,

$$
\begin{aligned}
M_{C M}(t) \vec{A}_{C M}(t) & =\frac{d \vec{P}(t)}{d t}+\frac{d^{2} m_{N}(t)}{d t^{2}}\left[\vec{r}_{N}(t)-\vec{R}_{C M}(t)\right] \\
& +\frac{d m_{N}(t)}{d t}\left[\vec{v}_{N}(t)-\vec{V}_{C M}(t)\right]-\frac{d M_{C M}(t)}{d t} \vec{V}_{C M}(t),
\end{aligned}
$$

in which

$$
\vec{A}_{C M}(t)=\frac{d \vec{V}_{C M}(t)}{d t}
$$


is the acceleration of the CM.

As

$$
\frac{d \vec{P}(t)}{d t}=\sum_{1}^{N} \frac{d \vec{p}_{i}(t)}{d t}
$$

we may insert the equation of motion 85 of the $i$-th particle into 100 we obtain

$$
\begin{aligned}
M_{C M}(t) \vec{A}_{C M}(t) & =\sum_{i=1}^{N}\left[\sum_{\substack{j=1 \\
j \neq i}}^{N} \vec{F}_{j \rightarrow i}^{(i n t)}(t)+\vec{F}_{i}^{(e x t)}(t)\right]+\frac{d^{2} m_{N}(t)}{d t^{2}}\left[\vec{r}_{N}(t)-\vec{R}_{C M}(t)\right] \\
& +\frac{d m_{N}(t)}{d t}\left[\vec{v}_{N}(t)-\vec{V}_{C M}(t)\right]-\frac{d M_{C M}(t)}{d t} \vec{V}_{C M}(t) .
\end{aligned}
$$

As in (54), Newton's Third Law and a little algebra shows that the contribution of internal forces vanishes,

$$
\sum_{i=1}^{N} \sum_{\substack{j=1 \\ j \neq i}}^{N} \vec{F}_{j \rightarrow i}^{(i n t)}(t)=\overrightarrow{0}
$$

so that, for the case of particles with varying mass, internal forces do not contribute to the dynamics of the CM. Together with 92 , that leads to the equation of motion of the CM

$$
\begin{aligned}
& M_{C M}(t) \vec{A}_{C M}(t)=\sum_{1}^{N} \vec{F}_{i}^{(e x t)}(t) \\
& -\frac{d^{2} m_{N}(t)}{d t^{2}}\left(\vec{R}_{C M}(t)-\vec{r}_{N}(t)\right) \\
& -\frac{d m_{N}(t)}{d t}\left(2 \vec{V}_{C M}(t)-\vec{v}_{N}(t)\right) .
\end{aligned}
$$

One of the differences between the previous result 105 and (68) is the absence of the term $+\frac{d m_{N}(t)}{d t} \cdot \vec{v}_{N}(t)$. This difference happens because in this section the mass of the $N$-th particles is time dependent and the physical forces that act on it are responsible for the time variation of the product of the mass by the vector velocity. In subsection 2 the mass of the migration particles does not vary and the physical forces only drive the time dependence of the vector velocity of the particle.

If the mass $m_{N}(t)$ of the $N$-th particle is of the form

$$
m_{N}(t)=m_{N}^{(0)} f(t-\bar{t})+m_{N}^{(f)},
$$

in which $m_{N}^{(0)}$ and $m_{N}^{(f)}$ are constants and $f(t)$ is as in (5) (see Appendix B for a particular example of the transition function $f(t)$ ), then the extra terms of the equation of motion 105 are non-vanishing only during the transition interval, for which

$$
\frac{d m_{N}(t)}{d t} \neq 0
$$

and

$$
\frac{d^{2} m_{N}(t)}{d t^{2}} \neq 0
$$

The term linear in $\left(\vec{R}_{C M}(t)-\vec{r}_{N}(t)\right)$ on the r.h.s. 105 of could be interpreted as a repelling or restoring force, either pulling the CM away from or pushing it towards its "normal" position, according to the instantaneous sign of $\frac{d^{2} m_{N}(t)}{d t^{2}}$. The term in 105 proportional to $\left(2 \vec{V}_{C M}(t)-\vec{v}_{N}(t)\right)$ could be interpreted as a damping force acting on the CM. Notice that this term differs from its correspondent one in Eq. (68).

The solution of 105 would require the knowledge of $\vec{r}_{N}(t)$ and $\vec{m}_{N}(t)$ for all $t$. In section 4 we will exploit some simple numerical solutions for the unidimensional case of free particles.

\section{Examples: the CM of a set of three particles}

The simplest example of the behavior of the CM of subsystem left by a migrating particle is provided by considering a unidimensional system with three free particles. These three particles could be sliding over a horizontal air rail with negligible friction. The three particles leave from the same space point on the air rail, but with different velocities. Let us imagine that those particles have positions given by

$$
\begin{aligned}
x_{1}(t) & =t, \\
x_{2}(t) & =\frac{t}{2}, \\
x_{3}(t) & =-t .
\end{aligned}
$$

in convenient units of length and time. Let the subsystem $S_{1}$ be composed of permanent particles 1 and 3 , with masses $m_{1}=1$ and $m_{3}=2$, respectively, and the migrating particle be the particle 2 , with mass equal to $m_{2}=1$. All the three particles have constant mass. Let the transition function that describes the movement of the particle 2 out of the subsystem $S_{1}$ be $f(5-t)$, in which

$$
f(\tau)=\frac{1}{2}+\frac{1}{2} \tanh (2 \tau) .
$$

The transition occurs about the instant $\bar{t}=5$, with interval amplitude $\Delta t \approx 1$, as Fig. 2 shows. The variable $t$ is in some suitable unit of time.

The mass of the CM of the subsystem $S_{1}$, at each time $t$, is 


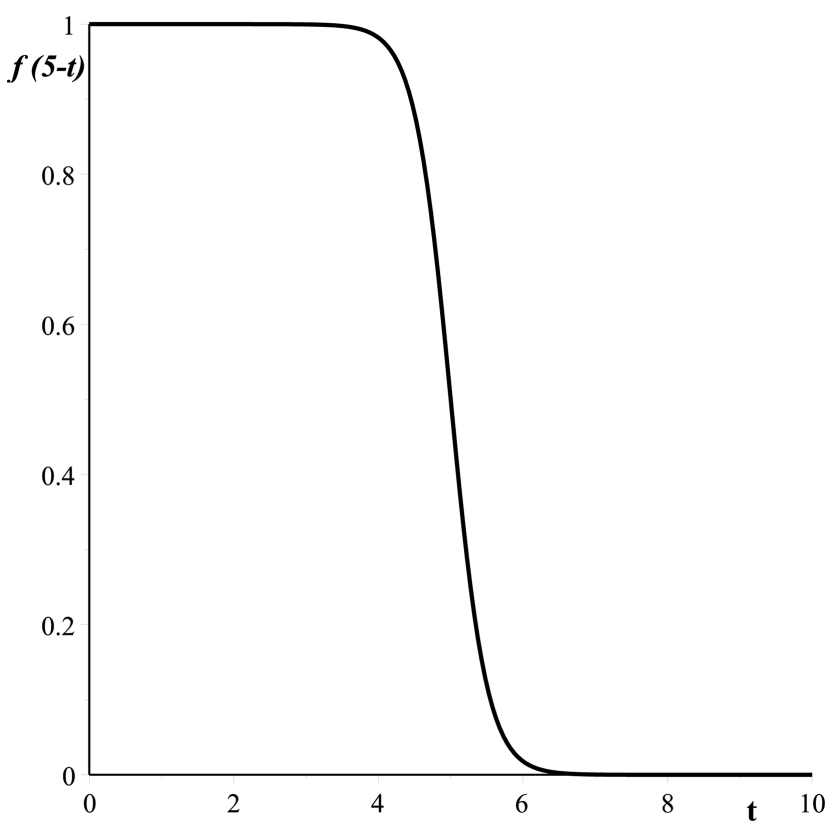

Figure 2: The transition function $f(5-t)$ that describes the location of the particle 2 at each moment $t$. During the time interval $t \in[4,6]$, approximately, the particle 2 leaves the subsystem $S_{1}$.

$$
\begin{aligned}
M_{1}(t) & =m_{1}+f(5-t) \cdot m_{2}+m_{3} \\
& =\frac{1}{2}(7+\tanh (2(5-t))) .
\end{aligned}
$$

The position of the CM of $S_{1}, X_{C M}^{(1)}(t)$, is

$$
\begin{aligned}
X_{C M}^{(1)}(t) & =\frac{m_{1} x_{1}(t)+f(5-t) \cdot m_{2} x_{2}(t)+m_{3} x_{3}(t)}{m_{1}+f(5-t) \cdot m_{2}+m_{3}} \\
& =\frac{t}{2} \times\left(\frac{\tanh (2(5-t))-3}{\tanh (2(5-t))+7}\right)
\end{aligned}
$$

Fig. 3 shows the value of the position of the three particles along the $x$-axis and that of the CM of the system of the particles that belong to the set $S_{1}$ at each time $t$, in the plane $(t, x)$. The curve of the CM of the particle set shows the position of the application of their total weight at instant $t$.

In the case discussed up to now, the masses of the three particles are constant. Applying Newton's Second Law, that drives the time evolution of theses particles, we obtain

$$
m_{i} \frac{d^{2} x_{i}(t)}{d t^{2}}=F_{R}^{(i)}(t), \quad i=1,2,3,
$$

where $F_{R}^{(i)}(t)$ is the resultant force that acts on the $i$-th particle at time $t$, and $\frac{d^{2} x_{i}(t)}{d t^{2}}=a_{i}(t)$, where $a_{i}(t)$ is the instant acceleration of that particle. From 109 - 111 we obtain

$$
\frac{d^{2} x_{i}(t)}{d t^{2}}=0, \quad i=1,2,3
$$

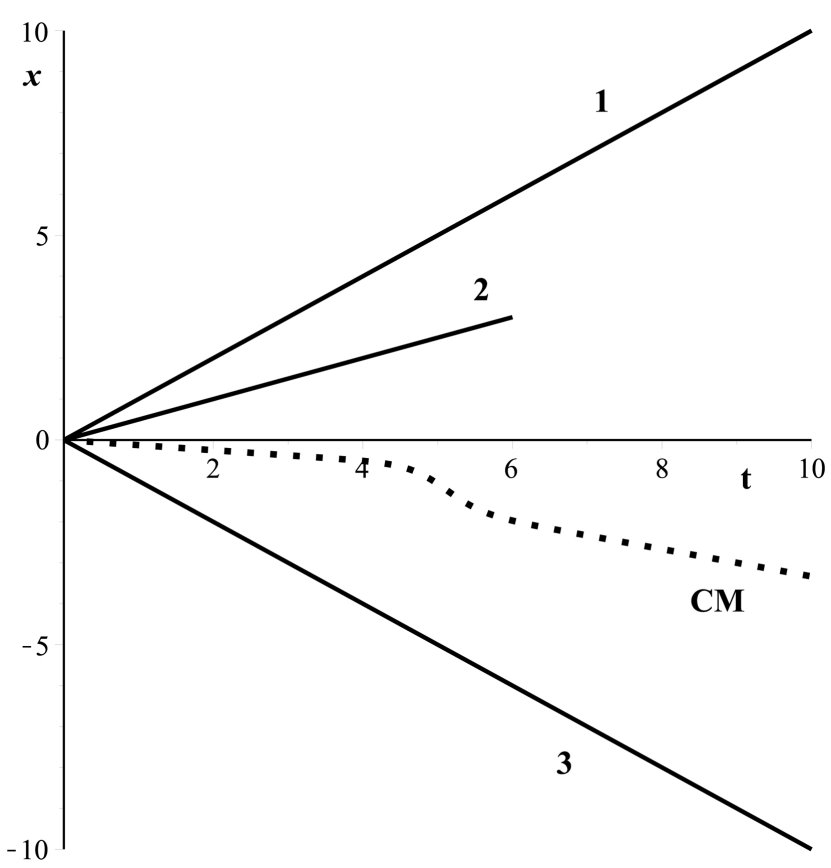

Figure 3: Positions of the 3 particles (solid lines) as a function of the time $t$ while they belong to the subsystem $S_{1}$, and of the CM of subsystem $S_{1}$ (dotted line), shown in the $(t, x)$ plane. The permanent particles are 1 and 3 ; as particle 2 leaves the system, the location of the $\mathrm{CM}$ as a function of time is also shown. The transition interval when the mass of particle 2 vanishes corresponds approximately to $t \in[4,6]$. For $t \gtrsim 6$, particle 2 does not belong to $S_{1}$ anymore.

showing that

$$
F_{R}^{(i)}(t)=0, \quad i=1,2,3
$$

at any instant $t$. Consequently the three mentioned particles are free particles with null resultant forces acting on each one of them. During the whole time interval $t \in[0,+\infty)$, the plot of the positions $x_{i}(t)$, for $i \in\{1,2,3\}$ in the $(t, x)$ plane is made of straight lines, as shown in Fig. 3 After $t=6$, particle 2 no longer belongs to the particle system that is under observation.

We apply (68), that drives the dynamics of the position of the CM of $S_{1}$, to derive the position of $X_{C M}^{(1)}(t)$ during the time interval $t \in(4,6)$, when $X_{C M}^{(1)}(t)$ does not follow a straight line. The acceleration of the CM of the subsystem $S_{1}, a_{C M}^{(1)}(t)$, is obtained from 68 , and in the absence of forces applied to the physical particles, see (117), it becomes, 


$$
M_{1}(t) a_{C M}^{(1)}(t)=-\left(\frac{d^{2} M_{1}(t)}{d t^{2}}\right) \cdot\left(X_{C M}(t)-x_{2}(t)\right)-2\left(\frac{d M_{1}(t)}{d t}\right) \cdot\left(V_{C M}(t)-v_{2}(t)\right),
$$

where $M_{1}(t)$ is given by $113, V_{C M}^{(1)}(t)$ is the velocity of the CM of the subset $S_{1}$,

$$
V_{C M}^{(1)}(t)=\frac{d X_{C M}^{(1)}(t)}{d t}=\frac{-1-20 t+4 \sinh (2(5-t)) \cosh (2(5-t))-20 \cosh ^{2}(2(5-t))}{2(7 \cosh (2(5-t))+\sinh (2(5-t)))^{2}} .
$$

We plot in Fig 4 the time dependence of the velocity of the $\mathrm{CM}$ of $S_{1}$ during the time interval $t \in[0,10]$. For $t \lesssim 4$ the velocity of the CM of $S_{1}$ is approximately constant, $V_{C M} \approx-0.125$, and for $t \gtrsim 6 V_{C M}^{(1)}(t)$, becomes approximately constant again, $V_{C M} \approx-0.333$, but with a different value from that of the beginning of the movement. During that time interval, the velocity $V_{C M}(t)$ varies in time, increasing its modulus for $t \in[4,5)$ and decreasing for $t \in(5,6)$ up to returning to a new constant value. Notice that the kinetic energy of the fictitious particle located at the CM is not conserved, although the physical particles that belong to the set are all free particles. Remember that we are creating the movement of a fictitious particle from the information of the physical particles but it does not mean that they have the same behaviour.

The expression of $x_{2}(t)$ is given by (110) and $v_{2}(t)$ is the instantaneous velocity of particle 2

$$
v_{2}(t)=\frac{d x_{2}(t)}{d t}=\frac{1}{2}
$$

From (113) and 1112 , we obtain

$$
\begin{aligned}
-\frac{d^{2} M_{1}(t)}{d t^{2}} & =-\frac{d^{2} f(5-t)}{d t^{2}} \\
& =4 \tanh (2(5-t)),
\end{aligned}
$$

and

$$
\begin{aligned}
-2 \frac{d M_{1}(t)}{d t} & =-2 \frac{d f(5-t)}{d t} \\
& =2 \operatorname{sech}(2(5-\mathrm{t})) .
\end{aligned}
$$

In Figs. 5 and 5 we plot the coefficients $-\frac{d^{2} M_{1}(t)}{d t^{2}}$ and $-2 \frac{d M_{1}(t)}{d t}$, respectively. From these plots we verify that the two terms on the r.h.s. on (118) are non-null in the interval $t \in(4,6)$. It is during this time interval that the movement of the CM of the subset $S_{1}$ does not describe a free particle with constant mass.

From Fig. 5 a we obtain that $-\frac{d^{2} M_{1}(t)}{d t^{2}}>0$ for $t \in$ $(4,5)$, such that the linear term $\left(X_{C M}(t)-x_{2}(t)\right)$ in
(118) is a repelling force during this interval of time, when the CM of this system leaves the first straight line for $t \lesssim 4$. This interpretation is compatible with Fig, 4 . since the modulus of the velocity of the $\mathrm{CM}$ of the system, $\left|V_{C M}(t)\right|$, increases during this interval of time. For the time interval $t \in(5,6), \mathrm{Fig} 5 \mathrm{Fa}$ shows that $-\frac{d^{2} M_{1}(t)}{d t^{2}}<0$ and the linear term in 118 becomes a restoration force, whose coupling coefficient depends on time.

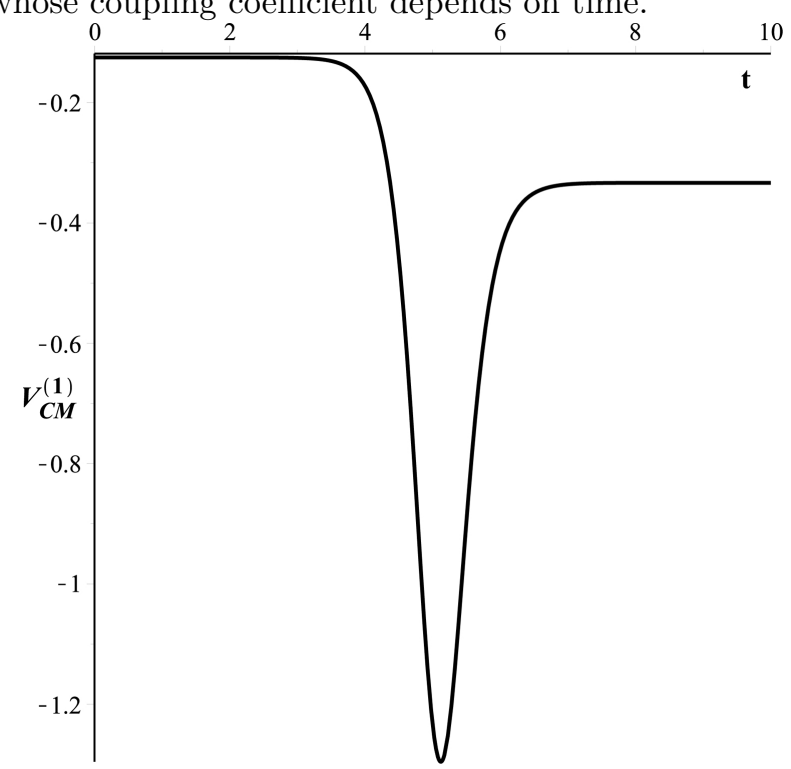

Figure 4: The time dependence of the velocity of the CM of subsystem $S_{1}, V_{C M}^{(1)}(t)$, during the time interval $t \in[0,10]$.

Fig $5 \mathrm{~b}$ shows that during the interval $t \in(4,6)$ we have $-2 \frac{d M_{1}(t)}{d t}>0$ and that the movement of the $\mathrm{CM}$ of $S_{1}$ is under the action of an effective dissipative force that decreases the modulus of its velocity, $\left|V_{C M}^{(1)}(t)\right|$. After $t \gtrsim 5$, when the effective linear force on the $\mathrm{CM}$ of $S_{1}$ becomes a restoring force, the modulus of $V_{C M}^{(1)}(t)$ diminishes.

The expression (114) can also give the location of the CM of a set of three particles, whose positions $x_{i}(t)$, with $i=1,2,3$, are given by eqs.109 - 1111, and with 

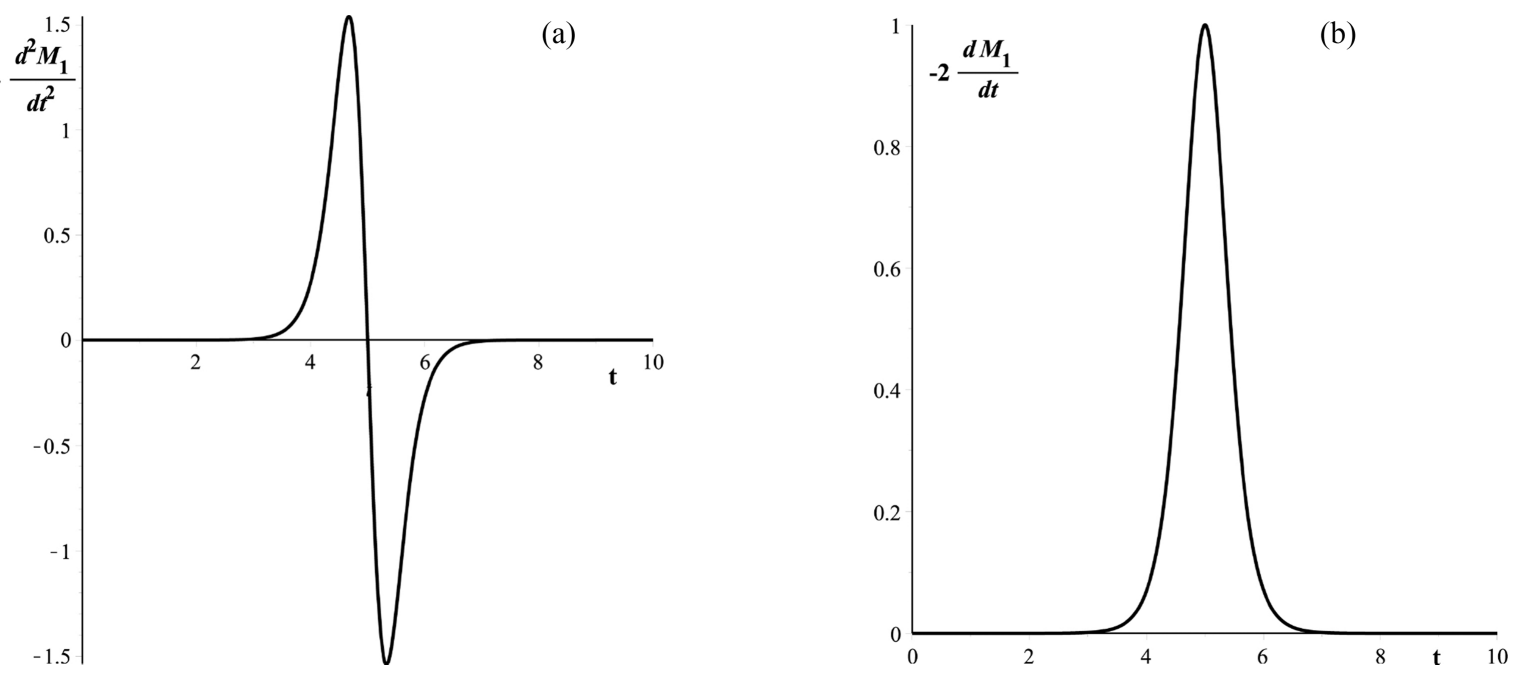

Figure 5: $a$ ) The plot of the coefficient $-\frac{d^{2} M_{1}(t)}{d t^{2}}$ as a function of time $t$. The expression of this coefficient is given by $(121) . b$ ) The plot of the coefficient $-2 \frac{d M_{1}(t)}{d t}$ as a function of time $t$, see 122 .

respective masses

$$
\begin{aligned}
m_{1} & =1, \\
m_{2}(t) & =\frac{1}{2}+\frac{1}{2} \tanh (2(5-t)), \\
m_{3} & =2 .
\end{aligned}
$$

The plot of $m_{2}(t)$ coincides with the curve $f(2(5-t))$ shown in Fig 2. The positions of these three particles, and the CM of this set of particles, are shown in Fig 3

In section 3 we pointed out that Eq. 105, that drives the dynamics of the CM of the system when the mass of one of its particles varies in time, differs from Eq. (68), that determines the instantaneous acceleration of the CM of a system with a migrating particle. How is it possible that the position of the CM of the subsystem $S_{1}$ with a migrating particle 2 , discussed previously, be the same as the CM of a set of the three particles with masses 123 - 125 that follow the paths 109 - 111)?

Let us discuss in detail the movement of the three particles that follow the positions $(109)$ - (111), whose masses are given by 123 - 125 . We call system $S$ this set of three particles. Newton's Second Law written for the particles 1 and 3 reads

$$
m_{i} \frac{d^{2} x_{i}(t)}{d t^{2}}=F_{R}^{(i)}(t), \quad i=1,3 .
$$

Form 109 and (111), we obtain

$$
F_{R}^{(i)}(t)=0, \quad i=1,3 ;
$$

that means that these two particles are free particles during the whole movement. The dynamics of particle 2 , whose mass varies in time, see (124), is

$$
\frac{d\left(m_{2}(t) v_{2}(t)\right)}{d t}=F_{R}^{(2)}(t)
$$

where from 110 we have

$$
v_{2}(t)=\frac{d x_{2}(t)}{d t}=\frac{1}{2}
$$

and $v_{2}$ has some suitable unit of velocity.

Substituting the value of $m_{2}(t)$, see (124), and the result 129 on the r.h.s. of 128 , we have

$$
\begin{aligned}
F_{R}^{(2)}(t) & =\frac{d}{d t}\left[\frac{1}{2}+\frac{1}{2} \tanh (2(5-t))\right] \\
& =\frac{1}{2} \frac{1}{\cosh ^{2}(2(5-t))} \Rightarrow \\
\Rightarrow \quad F_{R}^{(2)}(t) & =-\frac{1}{2} \operatorname{sech}^{2}(2(5-\mathrm{t})),
\end{aligned}
$$

From Fig 6 we verify that during the time interval $t \in(4,6)$, the force that acts on particle 2 is non null and the movement of this particles in this time interval is not of a free particle. The force $F_{R}^{(2)}(t)$ is responsible for the particle 2 to maintain a constant velocity, see $(120$, including the time interval $(4,6)$ when its mass is varying in time. Outside this time interval the movement of particle 2 coincides with that of a free particle.

The sum of the masses 123 - 125 gives the mass of the CM of system $S$, that is

$$
\begin{aligned}
M_{C M}(t) & =m_{1}+m_{2}(t)+m_{3} \\
& =\frac{1}{2}(7+\tanh (2(5-t))) \\
& =M_{1}(t)
\end{aligned}
$$

The dynamics of the CM of the system $S$ is given by (105), that in the present case becomes 


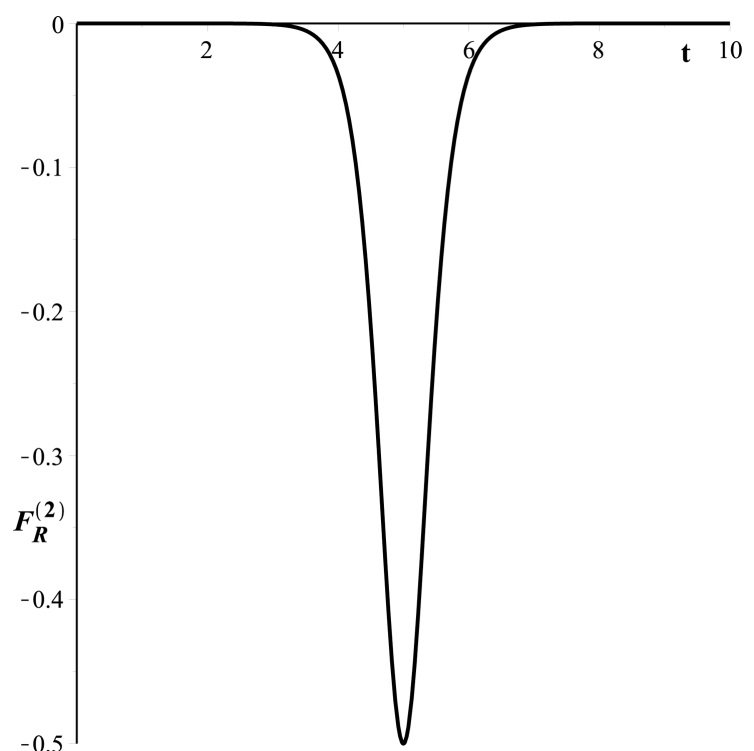

Figure 6: The curve $F_{R}^{(2)}(t)$ as a function of the time $t . F_{R}^{(2)}(t)$ is the force that acts on particle 2 during its movement.

$$
\begin{aligned}
& M_{C M}(t) A_{C M}(t)=F_{R}^{(2)}(t)-\frac{d^{2} m_{2}(t)}{d t^{2}} \\
& \times\left[X_{C M}(t)-x_{2}(t)\right]-\frac{d m_{2}(t)}{d t}\left[2 V_{C M}(t)-v_{2}\right]
\end{aligned}
$$

From (124) we obtain

$$
\begin{aligned}
-\frac{d^{2} m_{2}(t)}{d t^{2}} & =4 \tanh (2(5-t)) \\
-\frac{d m_{2}(t)}{d t} & =\operatorname{sech}^{2}(2(5-7)) .
\end{aligned}
$$

Note that $F_{R}^{(2)}(t)$, see 128 , can also be written as

$$
F_{R}^{(2)}(t)=\frac{d m_{2}(t)}{d t} v_{2}
$$

that being substituted into the r.h.s. of 132 yields

$$
\begin{aligned}
& M_{C M}(t) A_{C M}(t)=-\frac{d^{2} m_{2}(t)}{d t^{2}}\left[X_{C M}(t)-x_{2}(t)\right] \\
& -2 \frac{d m_{2}(t)}{d t}\left[V_{C M}(t)-v_{2}\right] .
\end{aligned}
$$

Since $M_{C M}(t)=M_{1}(t)$ and

$$
\begin{aligned}
\frac{d^{2} m_{2}(t)}{d t^{2}} & =\frac{d^{2} M_{1}(t)}{d t^{2}} \\
\frac{d m_{2}(t)}{d t} & =\frac{d M_{1}(t)}{d t}
\end{aligned}
$$

which shows that the differential equations (118) and (132) are identical, and the CM of the two systems of particles described in this paper follow the same trajectory.
Finally in Fig, 7 we compare the linear momentum of the the CM of the system $S, P_{C M}(t)$,

$$
P_{C M}(t) \equiv M_{C M}(t) V_{C M}(t),
$$

where $M_{C M}(t)$ and $V_{C M}(t)$ are given by (131) and (119), respectively, and the total linear momentum of the three particles, $P_{T}(t)$,

$$
P_{T}(t) \equiv m_{1} v_{1}+m_{2}(t) v_{2}+m_{3} v_{3},
$$

with $v_{i}(t)=\frac{d x_{i}(t)}{d t}, i=1,2,3$, and the positions $x_{i}(t)$ given by $109-111$.

From Fig, 7 we verify that for $t \lesssim 4$ and $t \gtrsim 6$ the momentum of the CM of $S, P_{C M}(t)$, and the total linear momentum of three particles that belong to $S, P_{T}(t)$, coincide. But during the interval $t \in[4,6]$ these two functions of linear momentum are quite distinct, as mentioned previously.

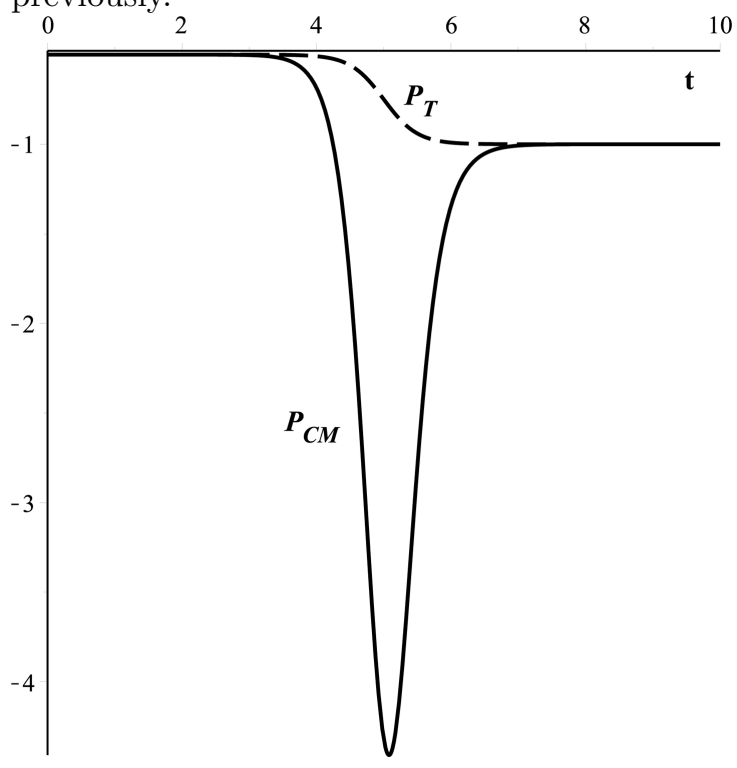

Figure 7: The curves of $P_{C M}(t)$ (solid line) of the CM of the system $S$ and $P_{T}(t)$ (dashed line), the total linear momentum of the three particles in the set $S$, in the interval $t \in[0,10]$.

The function $P_{C M}(t)$ and $P_{T}(t)$ coincide when the masses of the particles do not vary in time, or when the number of particles in the system is constant in time. We point out that during the whole movement the application of the total weight force of the particle set coincide with the position of $X_{C M}^{(1)}$.

\section{Conclusions}

A well-known result in the study of classical particle systems is that of the identity of the total momentum of a system and the momentum of the center of mass (CM), which is a fictitious particle with some defined properties to represent, in some sense, the system behavior. 
When the number of particles of a system or their masses vary in time, however, this identity may not hold true, at least during the time interval in which variations occur.

We have examined two situations. The first is that of a system composed of two subsystems with nonoverlapping permanent particles, but for a single particle that migrates from one subsystem to the other. We have obtained the equation of motion for the $\mathrm{CM}$ of one of the subsystems, showing that apart from the expected forces, other fictitious forces appear in the equation. Then we have studied the case of a single system in which one of its particles has a varying mass, obtaining a similar, but not identical, equation of motion for its CM. In both cases we have used smooth transition functions to describe continuous variations. The fictitious transient forces that appear are related not only to the time-dependence of the number/mass of particles, but also on the position and velocities of the CM, as well as on the migrating particle or variable-mass particle, analogous to instantaneous restoration and damping forces. We include in the presentation an example with three particles moving on an air rail, where we can describe the physical situation in terms of a migrating particle, or when the mass of one of the particles in the set varies in time.

Although we have extended the CM definition of a set of particles presented in Physics textbooks to include in this set a migration particle or a mass particle varying in time, the position where the total weight force of this system is applied coincides with that of our extended $\mathrm{CM}$ concept for these types of set.

It is interesting that students realize that they can raise new questions using the knowledge they have just learned in Newtonian Mechanics. Mainly they can find the answers to their own questions using the three Laws of Mechanics studied in the first classes of Physics.

\section{Supplementary material}

The following online material is available for this article: Appendix A - The position of the CM of a system of particles with variable masses

Appendix B - An explicit example for the transition function $f(t)$

\section{References}

[1] H.M. Nussenzweig, Curso de Física Básica (Edgar Blücher, São Paulo, 2002), $4^{\mathrm{a}}$ ed.

[2] J. Barcelos-Neto, Mecânica Newtoniana, Lagrangiana e Hamiltoniana (Ed. Livraria da Física, São Paulo, 2013), $2^{\mathrm{a}}$ ed .

[3] N.A. Lemos, Mecânica Analítica (Ed. Livraria da Física, São Paulo, 2004), $1^{\text {a }}$ ed.

[4] L.D. Landau and E.M. Lifshitz, Mechanics (ButterworthHeinenann, Oxford, 1976), $3^{\text {a }}$ ed.

[5] W. Greiner, Classical Mechanics, Systems of Particles and Hamiltonian Dynamics (Springer, New York, 2003).

[6] R.P. Feynman, R.B. Leighton and M. Sands, The Feynman Lectures on Physics (Addison-Wesley, Reading, 1965).

[7] W.T. Thomson, AIAA Journal 4, 766 (1966).

[8] B. Samardžija and S. Šegan, Publ. Astron. Obs. Belgrade 91, 97 (2012).

[9] F.O. Eke and T.C. Mao, Int. J. of Mechanical Engineering Education 30, 123 (2002).

[10] A. Nanjangud and F.O. Eke, Int. Rev. of Aerospace Engineering 5, 256 (2012). 